\title{
Liver infusion tryptose (LIT): the best choice for growth, viability, and infectivity of Leishmania infantum parasites
}

\author{
Ana Flávia Pereira Costa ${ }^{1}$. Rory Cristiane Fortes de Brito ${ }^{1} \cdot$ Lívia Mendes Carvalho $^{1}$ • \\ Jamille Mirelle de Oliveira Cardoso ${ }^{1}$ • Paula Melo de Abreu Vieira ${ }^{2,3}$. Alexandre Barbosa Reis ${ }^{1,4}$. \\ Rodrigo Dian de Oliveira Aguiar-Soares ${ }^{1} \cdot$ Bruno Mendes Roatt $^{1,3,4}$ (D)
}

Received: 13 May 2020 / Accepted: 16 September 2020 / Published online: 9 October 2020

(C) Springer-Verlag GmbH Germany, part of Springer Nature 2020

\begin{abstract}
Leishmania spp. parasites have a complex biological cycle presenting basically two different morphological stages, the amastigote and promastigote forms. In vitro cultivation allows a more complete study of the biological aspects of these parasites, indicating better conditions for infection, immunoassay tests, drug evaluations, and vaccines. Thus, we evaluated the three most used culture media for Leishmania spp., Grace's insect cell culture medium (Grace's), liver infusion tryptose (LIT), and Schneider's insect medium (Schneider's), without supplementation or supplemented with fetal calf serum (FCS) and bovine serum albumin (Albumin) to evaluate the growth, viability, and infectivity of the L. infantum promastigotes. It was observed that promastigote forms have a better growth in LIT and Schneider's with or without FCS when compared to that in Grace's. The supplementation with albumin promoted greater viability of the parasites independent of the medium. For in vitro infection of J774.A1 macrophages using light microscopy and flow cytometry analyses, FCS-supplemented LIT and Grace's promoted higher percentage of infected macrophages and parasite load compared with Schneider's media. Taken together, our results demonstrated that the supplementation of LIT culture medium with FCS is the most suitable strategy to cultivate Leishmania infantum parasites enabling the maintenance of growth and infective parasites for research uses.
\end{abstract}

Keywords Leishmania infantum · In vitro cultivation · Liver infusion tryptose · Grace's insect cell culture medium · Schneider's insect medium

Section Editor: Nawal Hijjawi

Electronic supplementary material The online version of this article (https://doi.org/10.1007/s00436-020-06893-z) contains supplementary material, which is available to authorized users.

Bruno Mendes Roatt

bmroatt@gmail.com; roatt@ufop.edu.br

1 Laboratório de Imunopatologia, Núcleo de Pesquisas em Ciências Biológicas/NUPEB, Universidade Federal de Ouro Preto, Morro do Cruzeiro, Ouro Preto, Minas Gerais 35400-000, Brazil

2 Laboratório de Morfopatologia, Núcleo de Pesquisas em Ciências Biológicas/NUPEB, Universidade Federal de Ouro Preto, Ouro Preto, Minas Gerais, Brazil

3 Departamento de Ciências Biológicas, Insituto de Ciências Exatas e Biológicas, Universidade Federal de Ouro Preto, Ouro Preto, Minas Gerais, Brazil

4 Instituto Nacional de Ciência e Tecnologia em Doenças Tropicais, INCT-DT, Salvador, Brazil

\section{Introduction}

Leishmania spp. parasites have a life cycle involving both a mammalian host and a vector insect (Lutzomyia and Phlebotomus genera) with different morphological aspects adapted to each host (Sunter and Gull 2017). Thus, these parasites can present themselves in two main morphological stages: extracellular promastigotes that sequentially evolve by undergoing several sub-developmental stages (procyclic, nectomonad, leptomonad, and metacyclic) in the invertebrate host, and intracellular amastigote forms present in the vertebrate hosts (Hommel 1999). The culture of Leishmania spp. is essential for the study of the biology of these parasites, and nowadays this area has been of great academic interest, playing an important role in understanding many aspects of the disease and hostparasite interaction (Santarem et al. 2014).

The promastigotes are the main form of the parasites isolated and cultivated in laboratory conditions. The main goal of in vitro cultivation of these protozoa parasites is to obtain an 
adequate number of alive, viable, and infective forms for research purposes. The actual capacity of grown promastigotes improves studies on antigens for diagnostic and vaccines production, in vitro and in vivo studies, and allows the determination of biological characteristics of the parasites and their sensitivity to leishmanicidal drugs (Rodrigues Ide et al. 2010; Visvesvara and Garcia 2002).

For Leishmania spp. promastigotes in vitro cultivation, NNN (McNeal, Novy \& Nicolle) is the first medium used to isolate the parasites (Nicolle 1908). On the other hand, when the purpose is the maintenance and growth, a liquid-type medium is routinely used including liver infusion tryptose (LIT), a classic medium for trypanosomatids. LIT was first used for Trypanosoma cruzi cultivation and Leishmania spp. have adapted very well to this medium. LIT is a serum- and blood-free medium containing a base of liver infusion broth, tryptose, and glucose (Sadigursky and Brodskyn 1986). Nowadays, many other axenic culture media are used for cultivation of Leishmania spp. parasites but two are more common in laboratory routine, Schneider's insect medium (Schneider's) and Grace's insect cell culture medium (Grace's). Both of them are defined media and are indicated for the continuous in vitro cultivation of promastigote forms of Leishmania (Merlen et al. 1999). However, all of them have in common the need for supplementation to ensure optimal parasite nutrition.

In this sense, fetal calf serum (FCS) is considered a key component of protein supplementation, mixture of purine bases and vitamins, which is used with success on axenic culture medium (Merlen et al. 1999). However, the careful control is necessary to obtain pathogen-free FCS as a requirement to prevent the spread of important zoonoses, such as brucellosis, mycoplasma infection, and "mad cow disease" (prion disease). In this way, bovine albumin fraction has been used as substitution of FCS and a supplement source to in vitro cultivation of Leishmania spp. parasites (Berens and Marr 1978; Sunter and Gull 2017). The most dangerous oversimplification problem working with alterations in the medium composition is to assume that all developed media will enable biologically identical promastigotes (Santarem et al. 2014). As a consequence, it is crucial to evaluate if media and supplementation could influence and modify promastigotes' biology.

For many years, studies have shown that parasite infectivity and virulence may vary according to the medium used for its cultivation (Moreira et al. 2012a; Smejkal et al. 1988). These studies were only possible due to the development of culture media that promoted the maintenance and growth of these parasites, which is fundamental for the use in infection studies and experimental challenge for drug and vaccine testing in vitro and in vivo models. However, great variability is observed in experimental assays, directly dependent on the culture medium and supplementation often impeding the reproducibility of studies in different laboratories (Rodrigues
Ide et al. 2010). Moreover, there are no studies in the literature that further evaluate Leishmania infantum parasites. Thus, the aim of this study was to compare the influence in the growth, morphology, viability, and in vitro infectivity of $L$. infantum promastigotes cultivated in different axenic media (Grace's, LIT, and Schneider's) with different supplements (FCS or bovine albumin), or without supplementation. This study will indicate the best medium composition to use in a laboratory routine to obtain rich and infectious cultures of Leishmania infantum parasites for research uses.

\section{Materials and methods}

\section{Parasites and axenic culture media}

The Leishmania infantum strain (MCAN/BR/2008/OP46) used in this work was isolated from a symptomatic dog from an endemic area of canine visceral leishmaniasis (Governador Valadares, Minas Gerais, Brazil). Restriction fragment length polymorphism-PCR analysis confirmed the species as $L$. infantum (Moreira et al. 2012b). The promastigote parasites were maintained at $23-24{ }^{\circ} \mathrm{C}$ in different culture media. The liver infusion tryptose (LIT) axenic medium was prepared according to Camargo (1964) with modifications. Briefly, a 4-salt solution ( $\mathrm{NaCl}, 40 \mathrm{~g} \mathrm{~L}^{-1} ; \mathrm{KCl}, 4 \mathrm{~g} \mathrm{~L}^{-1} ; \mathrm{Na}_{2} \mathrm{HPO}_{4}$, $80 \mathrm{~g} \mathrm{~L}^{-1}$; tryptose, $50 \mathrm{~g} \mathrm{~L}^{-1}$ all from Sigma) was prepared supplemented with bovine hemin $\left(0.25 \mathrm{~g} \mathrm{~L}^{-1}\right.$-Sigma $)$. After that, $50 \mathrm{~mL} \mathrm{~L}^{-1}$ of a $10 \%$ liver infusion broth (LIB - Gibco) solution and $10 \mathrm{~mL} \mathrm{~L}^{-1}$ of a $40 \%$ glucose solution (SigmaAldrich) were added. Grace's (Gibco) and Schneider's (Sigma-Aldrich) were prepared according to the manufacturer's instructions. Finally, 200 units $\mathrm{mL}^{-1}$ penicillin, 200 units $\mathrm{mL}^{-1}$ streptomycin, and 200 units $^{\mathrm{mL}^{-1}}$ neomycin were added and these solutions were stirred and filtered at $0.22 \mu \mathrm{m}$. To evaluate how the culture media supplementation could influence growth, viability, and infectivity of $L$. infantum promastigotes, Grace's, LIT, and Schneider's were used without supplementation, supplemented with $10 \%$ fraction $\mathrm{V}$ bovine albumin (Albumin), or supplemented with $10 \%$ fetal calf serum (FCS). The compositions of each media are represented in supplementary Table 1.

\section{Cultivation conditions and growth curve of $L$. infantum}

The cryopreserved $L$. infantum promastigotes were thawed directly in the axenic culture media (Grace's, LIT, and/or Schneider's) in the presence of NNN. Before starting the experiments, parasites were maintained in each medium with the different supplementation (not supplemented, supplemented with FCS, and supplemented with albumin) for a minimal of three passages. For the in vitro evaluation of 
parasite growth, promastigotes at the logarithmic phase were seeded at the density of $1 \times 10^{7}$ cells $\mathrm{mL}^{-1}$ to three different studied media (Grace's, LIT, and/or Schneider's) with different supplementations (10\% fetal calf serum and $10 \%$ fraction $\mathrm{V}$ bovine albumin) or non-supplemented. The cultivation of promastigotes was carried out in $25 \mathrm{~cm}^{2}$ plastic cell culture flasks containing $10 \mathrm{~mL}$ of each medium at $23-$ $24{ }^{\circ} \mathrm{C}$. The parasite count for growth curve was performed daily for 10 consecutive days. To do so, aliquots from different cultures were removed and diluted 1:20 in 5\% formaldehyde solution for parasite counting in a Neubauer chamber at $\times 400$ magnification under a light microscope (Zeiss AxioImager.Z2-Carl Zeiss, Germany). The experiments were performed in triplicate.

\section{Morphology and viability assessment}

During growth curves, on alternate days (odd days), the promastigote morphology analysis was performed. Aliquots of $50 \mu \mathrm{L}$ of each promastigote parasite culture were spread on glass slides. After drying at room temperature, the samples were fixed in methanol and stained by Giemsa. Morphological qualitative analyses of the promastigote forms were performed using a light microscope (Zeiss AxioImager.Z2 - Carl Zeiss, Germany) at $\times$ 400 magnification.

Moreover, L. infantum promastigote viability analysis was determined on the odd days of the growth curves using propidium iodide (PI-Thermo Fisher Scientific) labeling. For that, promastigote culture was washed and resuspended in a FACS tube (BD Biosciences) at a density of $10^{6} \mathrm{~mL}^{-1}$ in final volume of $200 \mu \mathrm{L}$ of PBS and $1 \mu \mathrm{L}$ of PI $\left(1 \mathrm{mg} \mathrm{mL}^{-1}\right)$ was added to mark dead cells. The samples were then incubated at room temperature for $15 \mathrm{~min}$. Parasite cultures subjected to $10 \%$ DMSO solution were used as a positive control of death. Data were collected in a BD FACSCalibur flow cytometer $\left(50,000\right.$ gated events) and analyzed by FlowJo ${ }^{\circledR}$ software (Becton Dickinson). This experiment was performed in triplicate.

\section{Promastigote staining using CFSE}

L. infantum promastigotes were prepared after Neubauer count in the respective stationary phase day. After that, the parasites were washed twice with PBS and $1 \times$ $10^{7}$ promastigotes $\mathrm{mL}^{-1}$ were labeled with $5 \mu \mathrm{M}$ of carboxyfluorescein succinimidyl ester (CFSE, Invitrogen) for $10 \mathrm{~min}$ at $37{ }^{\circ} \mathrm{C}$, shaken every $2 \mathrm{~min}$. Then, $9 \mathrm{~mL}$ of complete RPMI 1640 medium supplemented with $10 \%$ FCS was added and incubated for $10 \mathrm{~min}$ at room temperature. Then, tubes were centrifuged twice for $10 \mathrm{~min}$ at $1200 \mathrm{~g}$, the supernatant discarded, and the pellet homogenized. After the final centrifugation, the promastigotes were resuspended in complete RPMI 1640 medium before proceeding to macrophage infections, at final concentration of $1 \times 10^{7}$ parasites $\mathrm{mL}^{-1}$. The CFSE-stained parasites were used for all flow cytometry experiments.

\section{Macrophage culture and in vitro infection}

J774.A1 murine macrophages were maintained in culture flasks containing RPMI 1640 medium (pH 7.2-7.4) supplemented with $10 \%$ FCS, $2 \mathrm{mM} \mathrm{L-glutamine,} \mathrm{and}$ 200 units $\mathrm{mL}^{-1}$ penicillin, 200 units $\mathrm{mL}^{-1}$ streptomycin, and 200 units $\mathrm{mL}^{-1}$ neomycin (Sigma-Aldrich) in a humidified $5 \% \mathrm{CO}_{2}$ atmosphere at $37^{\circ} \mathrm{C}$. The macrophages were scraped using a cell scraper and the contents of the bottle were transferred to a falcon tube, which was centrifuged for $10 \mathrm{~min}$ at $1200 \mathrm{~g}$ at $4{ }^{\circ} \mathrm{C}$. After counting, cells were resuspended in RPMI with volume adjusted to $1 \times 10^{6}$ macrophages $\mathrm{mL}^{-1}$.

In vitro infection was performed in two different strategies. For light microscopy analyses, $10^{5}$ macrophages were added into chamber slide wells. After that, $5 \times 10^{5} \mathrm{~L}$. infantum promastigote forms in stationary growth phase of the respective culture media (1:5 ratio) were added. In flow cytometry evaluation, $10^{5}$ macrophages were added into polypropylene tubes. Similarly, $5 \times 10^{5} \mathrm{CFSE}$ staining L. infantum promastigote forms in stationary growth phase (1:5 ratio) were added. After $4 \mathrm{~h}$, non-internalized parasites were removed, and the cells were incubated for $48 \mathrm{~h}$ at $5 \% \mathrm{CO}_{2}$ and $37^{\circ} \mathrm{C}$. In all macrophage infection experiments, we selected the stationary phase day of $L$. infantum promastigotes (Table 1).

\section{In vitro infectivity evaluation by light microscopy and flow cytometry}

After $48 \mathrm{~h}$, the supernatant from each chamber slide well was discarded and the top of the plate removed. Then, each slide was fixed in methanol and stained by Giemsa. For infectivity evaluation, the slides were analyzed under an optical microscope (Olympus Optical, Japan) $\times 1000$ enlargement using immersion oil. A total count of 500 J774.A1 macrophages per well was performed, discriminating the number of infected and uninfected macrophages, as well as the number of

Table 1 Stationary phase day of L. infantum promastigotes in different axenic culture media (Grace's, LIT, and Schneider's) with different supplementations (Albumin or FCS) or non-supplemented

\begin{tabular}{llll}
\hline Supplementation & Grace's & LIT & Schneider's \\
\hline No supplementation (NS) & 4th day & 4th day & 3rd day \\
Albumin & 3rd day & 5th day & 5th day \\
Fetal calf serum (FCS) & 6th day & 4th day & 3rd day \\
\hline
\end{tabular}


amastigotes found within each infected macrophage. The results were expressed as follows:

- Percentage of infected macrophages $=$ No. of infected macrophages / total number of counted macrophages (infected and uninfected) $\times 100$;

- Average number of amastigotes $=$ ratio of the number of amastigotes per infected macrophage.

Similarly, the infection rate was evaluated by flow cytometry after $48 \mathrm{~h}$. The FACS tubes containing the infected macrophages were centrifuged for $10 \mathrm{~min}$ at $800 \mathrm{~g}$ at $4{ }^{\circ} \mathrm{C}$. The supernatant was discarded, and the tubes were washed twice using PBS under the same condition. Subsequently, the supernatant was discarded, and the cells were resuspended in $200 \mu \mathrm{L}$ of PBS for BD FACSCalibur flow cytometry reading. Data were obtained by the CellQuest ${ }^{\circledR}$ program, through the acquisition of 50,000 events per tube and analyzed using FlowJo ${ }^{\circledR}$ software (Becton Dickinson). The infection rate was obtained through the frequency of fluorescent J774.A1 macrophages. The mean fluorescence intensity (MFI) of the infected macrophages was calculated to indirectly measure the "parasitic load."

\section{Statistical analysis}

Statistical analyses were performed using Prism 7.0 software package (Prism Software, Irvine, CA, USA). The area under the curve (AUC) was performed to evaluate parasite growth in different media and supplements. In all analyses, the one-way ANOVA followed by Bonferroni's post-test for multiple comparisons was used when necessary. Differences were considered significant with $p<0.05$.

\section{Results}

\section{LIT supplemented with FCS promotes the best growth of $L$. infantum promastigotes}

When comparing the different supplements used in each axenic culture medium, we observed that FCS supplementation promotes the best results in the multiplication and growth rates of $L$. infantum promastigotes during the 10 days of evaluation in LIT and Schneider's media with higher results of area under curves $(p<0.05)$ when compared with the NS or Albumin supplementation (Fig. 1a).

Evaluating the different culture media in the absence of supplementation (NS), we observed that there was no difference in L. infantum promastigote growth during the 10 days of evaluation. On the other hand, when we used Albumin as supplement, we observed higher multiplication and growth rates $(p<0.05)$ in LIT medium compared with Grace's and Schneider's (Fig. 1b). In the same way, LIT medium showed higher multiplication and growth of $L$. infantum promastigotes $(p<0.05)$ compared with Grace's and Schneider's when FCS was used as supplement (Fig. 1b). Interestingly, the parasite growth from LIT and Schneider's displayed regular growth curves consisting of latent, exponential, stationary, and death phases, except those in Grace's medium (Fig. 1). The best growth curves were observed when FCS was used as supplement, and the NS and Albumin groups presented similar profiles in the growth of $L$. infantum promastigotes regardless of the medium used (Fig. 1).

\section{In vitro morphology characterization and viability of L. infantum promastigotes}

Microscopic examination indicated distinct promastigote forms identified without ambiguity. In general, two main distinct forms of promastigotes were observed, procyclic in the early days and metacyclic stages in the late days of growth (Sup. Fig. 1). Regarding Grace's medium, it was observed that the parasites were quite elongated and presented intense motility until the 5th day of evaluation (consistent with procyclic forms) (Sup. Fig. 1a). After the 5th day, the promastigotes became oval with gradual reduction of the parasite movement until the last day of the analysis (9th day). Morphologically, LIT and Schneider's presented similar aspects of promastigotes during the growth curve (Sup. Fig. 1). The promastigote forms in these two media were elongated with free flagella and intense movement dominant during the logarithmic phase. After 4-5 days of growth, it was observed that mixed forms with procyclic proportion decrease and increase metacyclic stages with intense motility and long flagellum with shorter/thinner body of the parasites (Sup. Fig. 1b and c). Moreover, it was observed that regardless of the medium, the use of albumin promoted a persistence of procyclic forms until 5-6 days of the growth curve (Sup. Fig. 1). After the 6th day, the procyclic forms became oval with gradual reduction of the movement until the last day of the analysis (9th day). In NS or FCS-supplemented media, a similar morphological aspect was observed mainly in Schneider's and LIT media. All described morphological aspects of the promastigotes are represented by arrows (Sup. Fig. 1). Regarding the viability, all three axenic media (Grace's, LIT, and Schneider's) presented similar results of viability with a higher number of viable promastigotes on day 1 and lower number on the 9th day (Fig. 2a). Evaluating the absence of supplementation (NS), we observed higher $(p<0.05)$ percentage of viable promastigotes in Grace's and LIT compared with Schneider's (Fig. 2b). In FCS supplementation, higher $(p<0.05)$ percentage of viable promastigotes was observed in Grace's medium compared with Schneider's medium (Fig. 2b). Concerning albumin supplementation, no differences were observed between the axenic media (Grace's, LIT, and Schneider's) (Fig. 2b). 


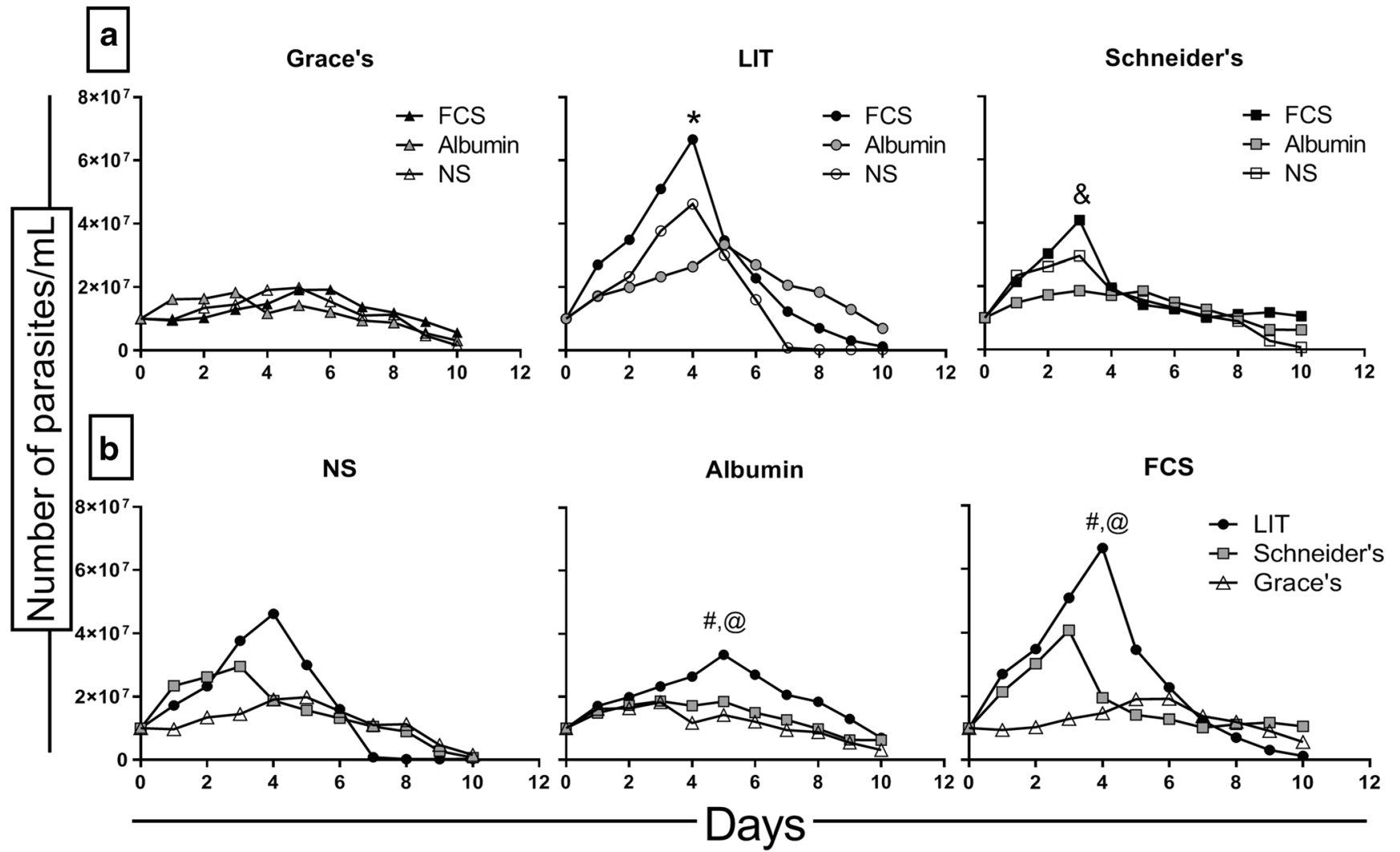

Fig. 1 Comparative analysis of the growth profile of L. infantum promastigotes (OP46 strain) cultivated in different axenic culture media for ten consecutive days. a Grace's, LIT, and Schneider's media. b Not supplemented (NS), albumin (Albumin), and fetal calf serum (FCS) supplementation. The initial inoculum was $1 \times 10^{7}$ promastigotes of $L$. infantum parasites. Significant differences $(p<0.05)$ are represented by the one-way ANOVA followed by Bonferroni's post-test of area under

Supplementary Table 2 summarizes the percentage of viability of promastigote forms throughout the follow-up of the growth curve in the different media and supplements.

\section{Promastigotes grown in LIT medium showed higher capacity of in vitro infectivities after FCS supplementation}

With the results obtained by the growth curves, we choose the day of the stationary growth phase of the L. infantum promastigotes of each medium as the ideal day for use in macrophage infections (Table 1). The major results of in vitro infection are shown in Figs. 3 and 4 representing the results obtained in microscopy and flow cytometry analysis, respectively.

Figure 3a illustrates the macrophage infection in LIT medium with different supplementations or non-supplemented. Illustrative image of LIT NS showed few infected macrophages with amastigotes (Fig. 3a-left panel). In the LIT Albumin (Fig. 3a-medium panel), more infected macrophages were observed compared with the NS group. In the the curve values. The symbols "**" and "\&" represent significant differences $(p<0.05)$ between FCS compared with NS and Albumin, respectively. The symbols “@”, and "\#”, represent significant differences $(p<0.05)$ between LIT compared with Grace's and Schneider's, respectively. Data are represented as means of three independent experiments

LIT FCS group, a higher number of infected macrophages and higher amastigote count were observed (Fig. 3a-right panel). Regarding Grace's medium, an increase $(p<0.05)$ in the percentage of infected macrophages in FCS supplementation compared with NS and Albumin supplementation (Fig. 3b) was observed. Similarly, the LIT medium showed an increase $(p<0.05)$ in the percentage of infected macrophages in FCS supplementation compared with the absence of supplementation (NS). Moreover, LIT supplemented with FCS showed higher $(p<0.05)$ percentage of infected macrophages compared with Grace's with the same supplementation (Fig. 3b). In Schneider's medium, an increase $(p<0.05)$ in the percentage of infected macrophages was observed in Albumin and FCS supplementation compared with NS. Furthermore, higher $(p<0.05)$ percentage of infected macrophages was observed in Schneider's supplemented with Albumin compared with that of Grace's with the same supplementation (Fig. 3b).

In addition to assessing the "parasite burden" inside the infected macrophages, we performed the count of amastigote forms. In general, it was observed that the supplementation with FCS, regardless of the culture medium employed, led to 
a

Grace's

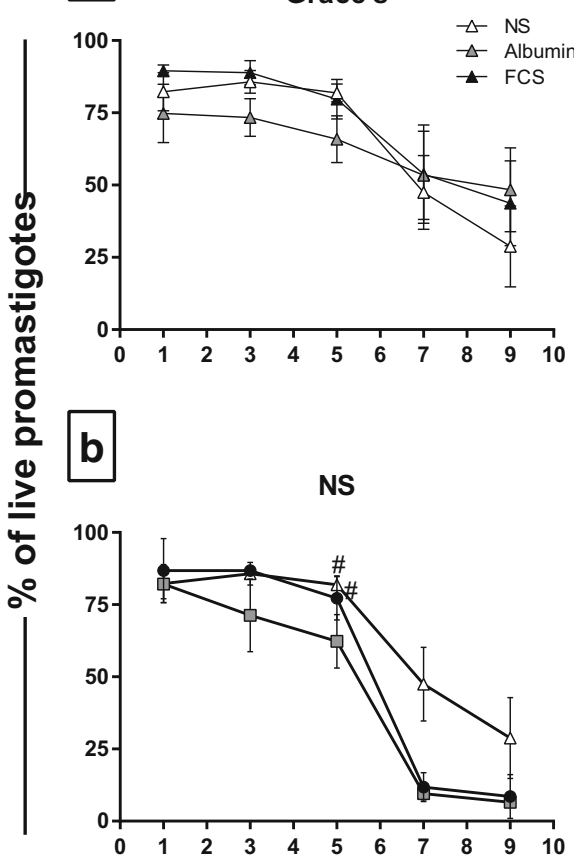

LIT

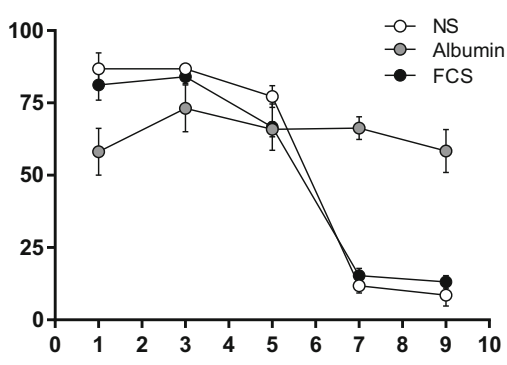

Albumin

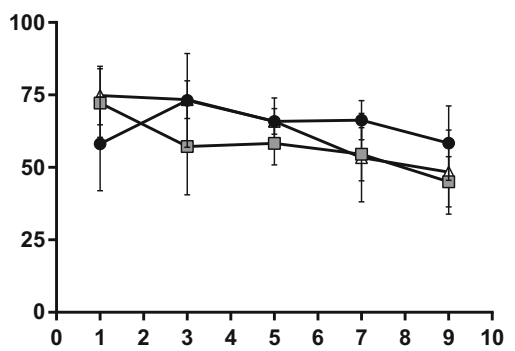

Schneider's

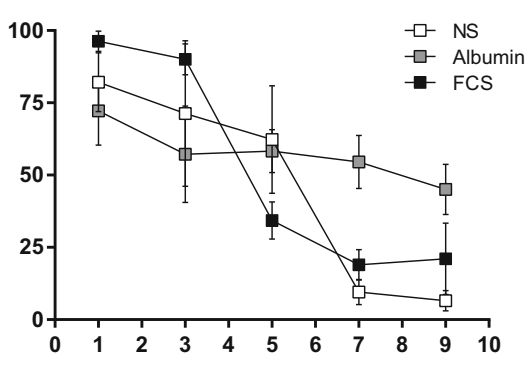

\section{Days}

Fig. 2 Comparative analysis of the viability profile of $L$. infantum promastigotes (OP46 strain) cultivated in different axenic culture media for ten consecutive days. a Grace's, LIT, and Schneider's media. b Not supplemented (NS), albumin (Albumin), and fetal calf serum (FCS) supplementation. The graphs represent the percentage of living

higher parasite load values compared with the other supplementation (Fig. 3c). In this sense, an increase $(p<0.05)$ in the parasite burden was observed in Grace's medium when we used FCS when compared with Albumin supplementation (Fig. 3c). The LIT medium showed an increase $(p<0.05)$ on the parasite load in FCS supplementation when compared with Albumin and NS. Moreover, NS showed an increase $(p<0.05)$ on the parasite burden in relation to Albumin (Fig. $3 c)$. Interestingly, the LIT supplemented with Albumin and FCS showed higher $(p<0.05)$ parasite burden compared with Grace's medium with the same supplementation (Fig. 3c). Furthermore, LIT showed an increase $(p<0.05)$ on the parasite load in all supplementations when compared with Schneider's medium (Fig. 3c). Likewise, Schneider showed an increase $(p<0.05)$ on the parasite load compared with Grace's when both media were supplemented with Albumin (Fig. 3c). The Schneider's medium showed an increase $(p<0.05)$ on the parasite load in NS and FCS supplementation when compared with Albumin (Fig. 3c). Evaluating the percentage of infected macrophages using flow cytometry, an increase $(p<0.05)$ in Albumin and FCS supplementation was observed in Grace's medium compared with NS (Fig. 4b). Moreover, FCS supplementation demonstrated promastigotes in odd days. Significant differences $(p<0.05)$ are represented by the one-way ANOVA followed by Bonferroni's posttest of area under the curve values. The symbol "\#” represents significant differences $(p<0.05)$ between Grace's and LIT compared with Schneider's

higher percentage $(p<0.05)$ of infected macrophages compared with Albumin (Fig. 4b). In addition, an increase $(p<0.05)$ in the percentage of infected macrophages was observed in the LIT supplemented with FCS compared with NS and Albumin (Fig. 4b). Similarly, an increase $(p<0.05)$ in the percentage of infected macrophages was observed in Schneider's NS and supplemented with FCS compared with Albumin. Moreover, FCS supplementation demonstrated higher percentage $(p<0.05)$ of infected macrophages compared with NS (Fig. 4b). Comparing the supplementation, we observed an increase $(p<0.05)$ in the percentage of infected macrophages in Schneider's NS in relation to Grace's and LIT NS. Interestingly, the LIT supplemented with FCS showed higher $(p<0.05)$ percentage of infected macrophages compared with Grace's and Schneider's media with the same supplementation (Fig. 4b).

In the same way, we performed a parasite burden evaluation using MFI values of infected macrophages. Grace's medium showed an increase $(p<0.05)$ on the parasite burden in Albumin and FCS supplementation compared with NS. Moreover, FCS supplementation demonstrated higher $(p<0.05)$ parasite burden compared with Albumin (Fig. 4c). In LIT medium, we observed an increase $(p<0.05)$ on the 
a
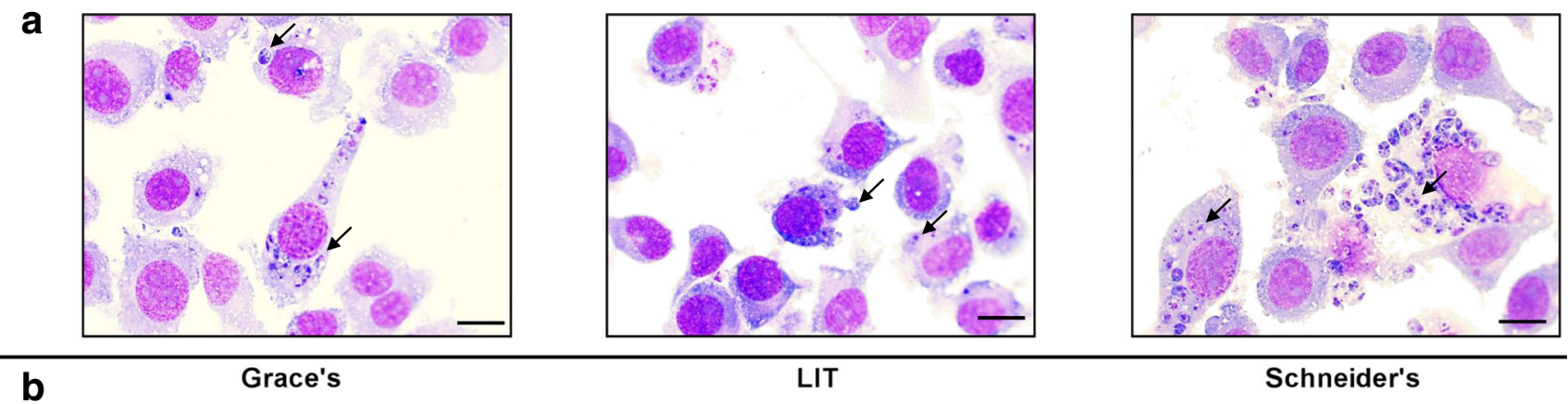

LIT

Schneider's
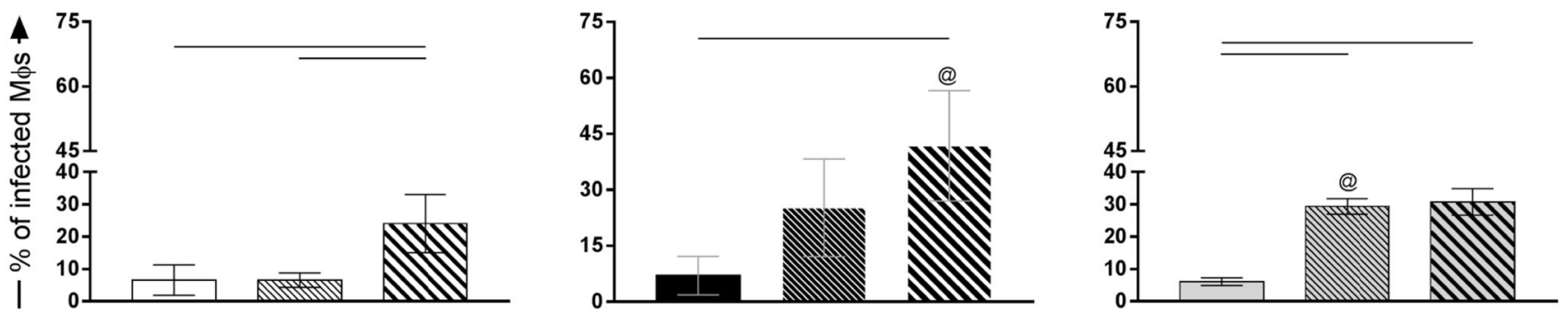

\section{C}
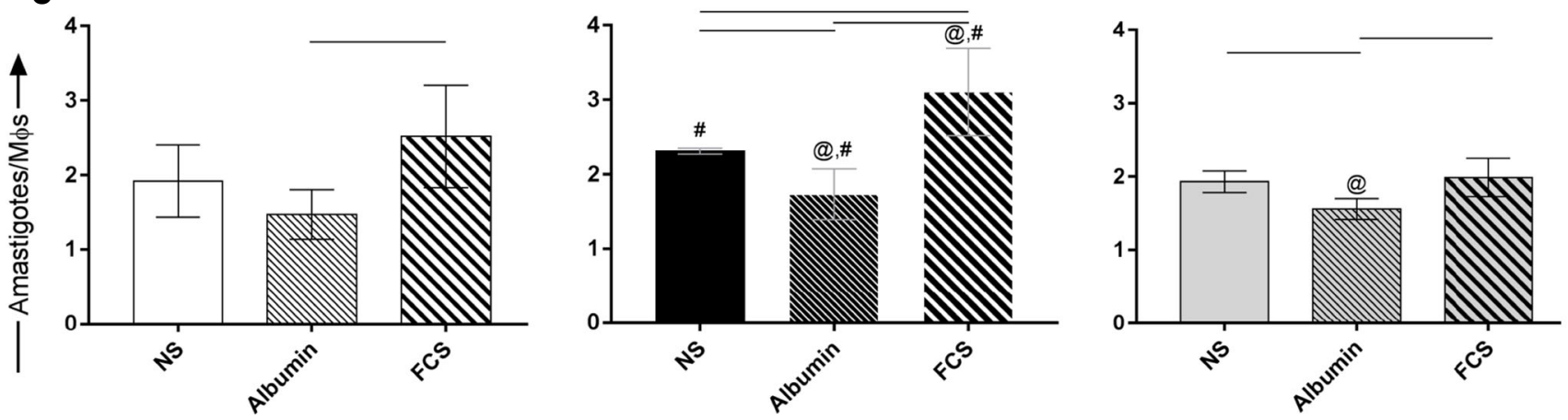

Fig. 3 Microscopy evaluation of J774.A1 murine macrophages infected with $L$. infantum promastigotes grown under different culture media (Grace's, LIT, or Schneider's) and different supplements (supplemented with albumin or FCS) or not supplemented. a Schematic images of light microscopic evaluation after $48 \mathrm{~h}$ of $L$. infantum macrophage infection cultured in LIT medium not supplemented-NS (left image), Albumin (medium image), and FCS (right image). b The percentage of infected

macrophages in different Grace's, LIT, or Schneider's culture media with different supplements (NS, albumin, or FCS). c The number of amastigotes in infected macrophages in Grace's, LIT, or Schneider's culture media with different supplements (NS, albumin, or FCS). The symbols “@” and “\#”" represent significant differences $(p<0.05)$ between LIT compared with Grace's and Schneider's. Data are represented as means \pm standard deviation (SD) of three independent experiments (triplicate)

parasite burden in the FCS compared with NS and Albumin supplementation (Fig. 4c). In addition, Schneider's supplemented with FCS showed an increase $(p<0.05)$ on the parasite burden compared with Albumin (Fig. 4c). Comparing the supplementation, we observed an increase $(p<0.05)$ on the parasite burden in Grace's FCS supplemented in relation to LIT and Schneider's (Fig. 4c). Interestingly, the LIT and Schneider's supplemented with Albumin showed higher $(p<0.05)$ parasite burden compared with Grace's on the same supplementation (Fig. 4c).

\section{Discussion}

The maintenance and cultivation of Leishmania promastigote forms, in general, seem not to be so problematic, as proven by the ability of these parasites to grow in many media, not to mention that most of these media were not developed for this group of pathogens. The related problems go beyond the simple cultivation, and the difficulties are related to the purpose of applying available media. The researchers should take into account the objective of their studies if the parasites are for laboratory maintenance, diagnosis, vaccine development, or generation of infective parasites (Jenson 2019).

In this context, different media for Leishmania cultivation have been proposed. The most described media in the literature are liver infusion tryptose (LIT) which is the oldest, classical, and complex undefined medium. On the other hand, Grace's and Schneider's are defined media, easy to prepare and use. There is a lack of standardization regarding the use of media for different purposes of parasite cultivation. In this sense, our study aimed to compare the three most 
a

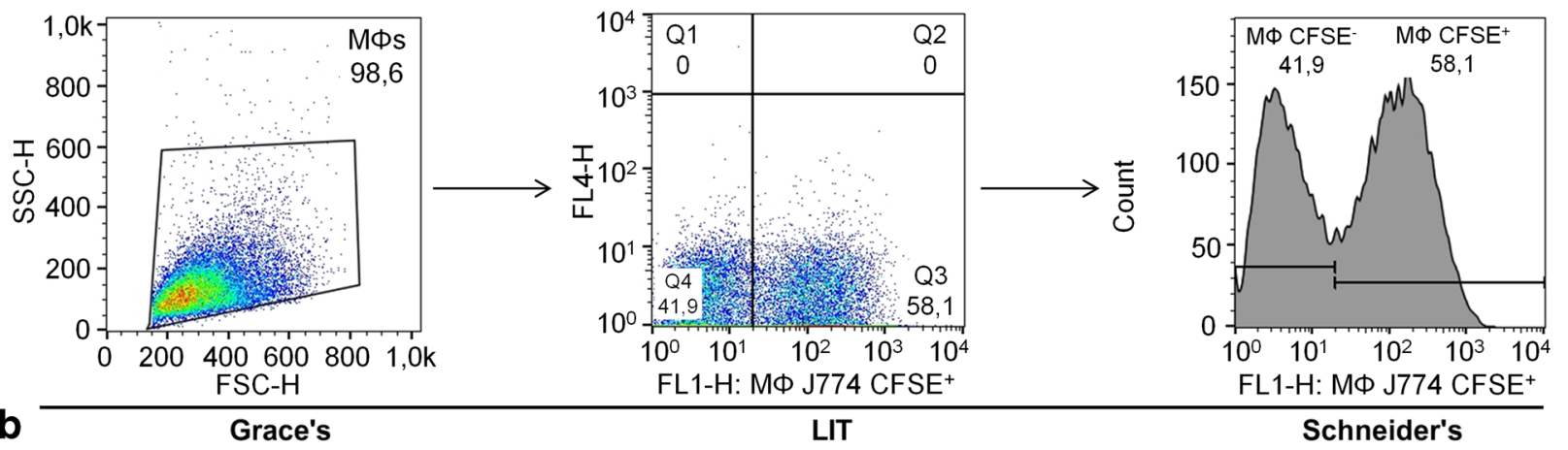

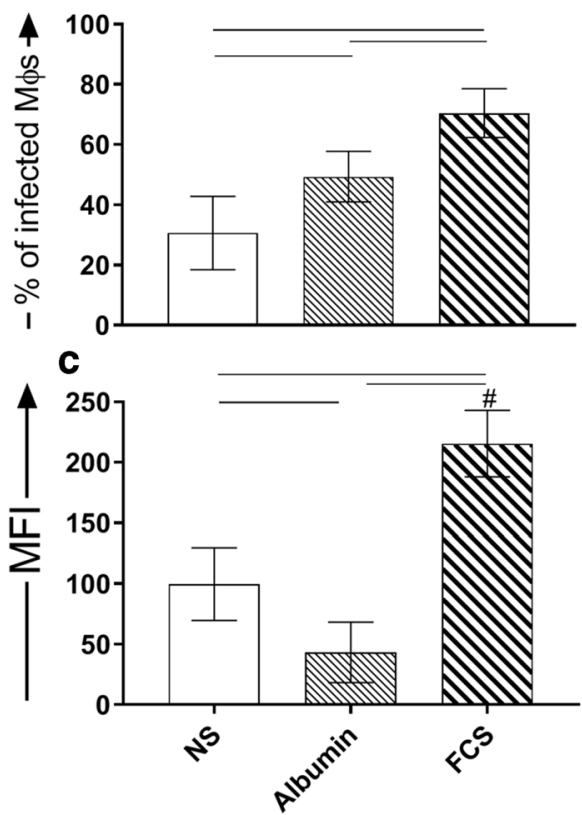

Fig. 4 Frequency evaluation of J774.A1 murine macrophages infected by L. infantum promastigotes grown under different culture media (Grace's, LIT, or Schneider's) and different supplements (NS and supplemented with albumin or FCS) assessed by flow cytometry. a The analysis strategy used for the evaluation of infection rate of amastigote forms of the $L$. infantum parasite in J774.A1 macrophages. b The percentage of infected macrophages in different Grace's, LIT, or Schneider's culture

used media for Leishmania cultivation with different supplementation and to identify the best of them that could promote better growth, improve the viability, and enhance the infectivity of $L$. infantum promastigote forms. To do so, we evaluated two commercial media, Grace's and Schneider's, that are chemically defined, which means they have in their formulation known and quantified components, e.g., amino acids, vitamins, and inorganic salts. The main difference between these media is that Schneider's has yeast extract. We also evaluated the LIT medium, which is classically used for trypanosomatid cultivation (mainly Leishmania spp. and Trypanosoma cruzi), but the exact composition of this medium is not well established due to the variety of compounds that are present in its formulation (Camargo 1964; Francis 2010). Furthermore, we evaluated the effect of medium supplementation adding fetal calf serum (FCS) and bovine serum albumin (Albumin). FCS is classified as a universal supplement because it allows the growth of many cell types (Brunner et al. 2010; Gstraunthaler 2003). Albumin has been reported to be involved in the preparation of various media improving the successful cultivation of mammalian cells (research and commercial fields) (Francis 2010).

Regarding parasite growth, we observed that LIT and Schneider's, supplemented or not, promoted a similar growth curve in L. infantum promastigotes, and they are better for parasite multiplication when compared with Grace's. This fact may be due to the composition of the media, since LIT has hemin, which is the source of heme, an essential compound for continuous growth and multiplication of promastigotes, and it also has liver infusion broth (LIB) that provides extra 
nucleoside sources (Merlen et al. 1999). The performance of Schneider's is related to the presence of yeast extract, which is a major stimulator of growth in axenic cultures, and it can be a fundamental source of nucleic acids (Barbosa et al. 2018). On the other hand, Grace's medium is not an important source of nucleoside, mainly adenosine; thus, it provided lower parasite growth (Francis 2010). Mahamoud et al. (2013) observed that the cultivation of $L$. donovani in LIT had a quick multiplication achieving the exponential phase around 10 days. In another study, the same Leishmania species was cultivated in Schneider's, and once again, this medium was able to promote rapid parasite growth (Dey et al. 2002). As observed in our results, a study conducted by Ali et al. (1998) evaluated an axenic culture medium, AJM-1, which is a Grace's-like medium and they observed a low yield in the L. major multiplication. We observed that when we added different supplementation to the Grace's media, there were no changes in the parasite morphology until the 5th day, after which we observed some oval forms in the culture except for the media supplemented with albumin. The morphological alterations are expected since, as described in the literature, there is glucose consumption which leads to a $\mathrm{pH}$ increase affecting the promastigote walls (Fritsche et al. 2007). In different circumstances, using the supplements interferes directly with the viability of the promastigotes. The presence of FCS in the media promotes greater viability when compared with non-supplemented. This finding could be explained by the features of FCS which is a complex mixture of various constituents, lower and higher weight biomolecules with a balance of promoters and inhibitors of growth (Gstraunthaler 2003).

In general, FCS is universally used to a massive proliferation of the parasites and differs from purified albumin that presents a lower ability to stimulate parasite replication as we observed. On the other hand, our data demonstrated high parasite viability in the media supplemented with albumin. This corroborates with various studies that reported the beneficial effect of albumin in mammalian cell viability due to the antioxidant properties, capability to bind to a wide range of ligands, stimulation of pinocytosis, late-onset apoptosis, and nutritional factor (Ellmerer et al. 2000; Erkan et al. 2005; Francis 2010; Peters 1996; Steiger and Steiger 1977). Furthermore, the high viability found in Grace's medium can be explained by the lower number of promastigotes that leads to minor nutrient exhaustion, e.g., glucose, fundamental for parasite growth (Fritsche et al. 2007).

Regarding the in vitro infectivity of the parasite, we found a higher agreement between the parasite load evaluated by optical microscopy and flow cytometry observing more infective promastigotes in the FCS-supplemented cultures. This result was expected taking into account most articles involving in vitro and in vivo Leishmania infection studies that describe the use of FCS supplementation (de Brito et al. 2019; Santarem et al. 2014; Siripattanapipong et al. 2019). The removal of the FCS leads to the loss of proteins, nucleoside sources, and hemoglobin, directly affecting the growth and infectivity of the promastigotes (Carvalho et al. 2009; Krishnamurthy et al. 2005). Surprisingly, the $L$. infantum promastigotes cultivated in Grace's medium demonstrated a lower percentage of infected macrophages but high parasite load (as shown by optical microscopy and flow cytometry analysis). This can be explained by the nature of the medium which is largely used in many studies to induce infection in animal models (da Silva Jr. et al. 2015; Silva et al. 2018; Siripattanapipong et al. 2019). Based on our data, we can suggest that Grace's medium, instead of inducing procyclic promastigotes, probably induces the formation of metacyclic promastigotes that explains the high parasite load in the infected macrophages in vitro. The $L$. infantum promastigotes cultivated in Schneider's medium presented a lower in vitro capacity to infect macrophages confirming the findings of Santarem's study that demonstrated an atypical behavior of $L$. infantum promastigotes cultivated in this medium (Santarem et al. 2014). The parasite profile suggested continuous division with the absence of a defined stationary phase and the presence of procyclic promastigotes. Moreover, there was a possibility of Schneider's-grown parasites failing to originate a large number of metacyclic forms (Santarem et al. 2014). The L. infantum promastigotes cultivated in LIT medium showed to be more infective in vitro for macrophages and exhibited high parasite load in the infected cells. Many studies have reported the use of this medium to promote metacyclogenesis in Trypanosoma spp. (Fajardo et al. 2016) but it seems to be successful for Leishmania species. Reports of infection of dogs (Roatt et al. 2012), hamsters (Moreira et al. 2012b), mice (Reis et al. 2017), and sand flies (Agrela and Feliciangeli 2015) demonstrate the potential use of this medium concerning in vivo Leishmania infection.

The different growth, viability, and in vitro infection profiles of $L$. infantum cultivated in different supplemented media presented in this study revealed that the choice of a specific medium and supplementation depends on the purpose of the study. For example, Grace's supplemented with FCS, the medium that provided prolonged cell viability, would be suitable for long-term cultivation. Regarding parasite mass production, e.g., aiming for DNA extraction, LIT or Schneider's supplemented with FCS would be an excellent choice. For effective in vitro infections concerning drug tests, treatment studies, and vaccine development, LIT and Grace's with FCS should be the best choice.

\section{Conclusions}

In conclusion, this article is the first study to compare the three classical media (with different supplementation) for Leishmania infantum promastigote cultivation according to 
their ability to promote growth, viability, and infectivity of in vitro macrophages. This study has demonstrated that there is a high influence between the media and supplementation promoting specific characteristics of the culture media. It would benefit various aspects of the next research studies concerning this key pathogenic parasite. Moreover, we conclude that the supplementation of LIT culture medium with FCS is the most suitable strategy to cultivate these parasites enabling the maintenance of growth and infective parasites for research uses.

Funding The authors acknowledge the Brazilian agencies CNPq (grant number 435224/2018-2), FAPEMIG (grant number APQ-03505-13 PROGRAMA DE PESQUISA PARA O SUS-PPSUS, APQ-01373-14 - PRONEX and APQ-02556-18, APQ-02577-18), CAPES (this study was financed in part by the Coordenação de Aperfeiçoamento de Pessoal de Nível Superior-Brasil (CAPES) - Finance Code 001), and Universidade Federal de Ouro Preto - UFOP for funding. R.C.F.B. is grateful for CAPES fellowship, and A.B.R. and B.M.R. are also grateful to $\mathrm{CNPq}$ for fellowships.

Data availability The datasets generated during and/or analyzed during the current study are available from the corresponding author upon reasonable request.

Compliance with ethical standards The Leishmania infantum OP46 strain used in this study has approval from the National System for the Management of Genetic Heritage and Associated Traditional Knowledge-SISGen (A55DE5A).

Conflict of interest The authors declare that they have no conflict of interest.

\section{References}

Agrela IF, Feliciangeli MD (2015) Effect of Leishmania spp infection on the survival, life expectancy, fecundity and fertility of Lutzomyia longipalpiss.1. and Lutzomyia pseudolongipalpis. Mem Inst Oswaldo Cruz 110:611-617. https://doi.org/10.1590/007402760150064

Ali SA, Iqbal J, Ahmad B, Masoom M (1998) A semisynthetic fetal calf serum-free liquid medium for in vitro cultivation of Leishmania promastigotes. Am J Trop Med Hyg 59:163-165. https://doi.org/ 10.4269/ajtmh.1998.59.163

Barbosa ADS, Cardozo ML, Dib LV, Fonseca ABM, Uchoa CMA, Bastos OMP, Amendoeira MRR (2018) Comparative study of three xenic media culture for cultivation of Balantidium coli strains. Rev Bras Parasitol Vet 27:20-26. https://doi.org/10.1590/S198429612017075

Berens RL, Marr JJ (1978) An easily prepared defined medium for cultivation of Leishmania donovani promastigotes. J Parasitol 64:160

Brunner D, Frank J, Appl H, Schoffl H, Pfaller W, Gstraunthaler G (2010) Serum-free cell culture: the serum-free media interactive online database. ALTEX 27:53-62. https://doi.org/10.14573/altex. 2010.1.53

Camargo EP (1964) Growth and differentiation in Trypanosoma cruzi. I. Origin of metacyclic trypanosomes in liquid media. Rev Inst Med Trop Sao Paulo 6:93-100

Carvalho S, Cruz T, Santarém N, Castro H, Costa V, Tomás AM (2009) Heme as a source of iron to Leishmania infantum amastigotes. Acta
Trop 109:131-135. https://doi.org/10.1016/j.actatropica.2008.10. 007

da Silva IA Jr, Morato CI, Quixabeira VB, Pereira LI, Dorta ML, de Oliveira MA, Horta MF, Ribeiro-Dias F (2015) In vitro metacyclogenesis of Leishmania (Viannia) braziliensis and Leishmania (Leishmania) amazonensis clinical field isolates, as evaluated by morphology, complement resistance, and infectivity to human macrophages. Biomed Res Int 2015:393049-393015. https://doi.org/10.1155/2015/393049

de Brito RCF, Cardoso JMO, Reis LES, Mathias FAS, Aguiar-Soares RDO, Teixeira-Carvalho A, Roatt BM, Correa-Oliveira R, Ruiz JC, Resende DM, Reis AB (2019) Synthetic peptides elicit strong cellular immunity in visceral leishmaniasis natural reservoir and contribute to long-lasting polyfunctional T-cells in BALB/c mice. Vaccines (Basel) 7. https://doi.org/10.3390/vaccines7040162

Dey T, Afrin F, Anam K, Ali N (2002) Infectivity and virulence of Leishmania donovani promastigotes: a role for media, source, and strain of parasite. J Eukaryot Microbiol 49:270-274. https://doi.org/ 10.1111/j.1550-7408.2002.tb00369.x

Ellmerer M, Schaupp L, Brunner GA, Sendlhofer G, Wutte A, Wach P, Pieber TR (2000) Measurement of interstitial albumin in human skeletal muscle and adipose tissue by open-flow microperfusion. Am J Physiol Endocrinol Metab 278:E352-E356. https://doi.org/ 10.1152/ajpendo.2000.278.2.E352

Erkan E, Devarajan P, Schwartz GJ (2005) Apoptotic response to albumin overload: proximal vs. distal/collecting tubule cells. Am J Nephrol 25:121-131. https://doi.org/10.1159/000084888

Fajardo EF, Cabrine-Santos M, Ferreira KA, Lages-Silva E, Ramirez LE, Pedrosa AL (2016) Semisolid liver infusion tryptose supplemented with human urine allows growth and isolation of Trypanosoma cruzi and Trypanosoma rangeli clonal lineages. Rev Soc Bras Med Trop 49:369-372. https://doi.org/10.1590/0037-8682-0190-2015

Francis GL (2010) Albumin and mammalian cell culture: implications for biotechnology applications. Cytotechnology 62:1-16. https://doi. org/10.1007/s10616-010-9263-3

Fritsche C, Sitz M, Weiland N, Breitling R, Pohl HD (2007) Characterization of the growth behavior of Leishmania tarentolae: a new expression system for recombinant proteins. J Basic Microbiol 47:384-393. https://doi.org/10.1002/jobm.200710111

Gstraunthaler G (2003) Alternatives to the use of fetal bovine serum: serum-free cell culture. ALTEX 20:275-281

Hommel M (1999) Visceral leishmaniasis: biology of the parasite. J Inf Secur 39:101-111. https://doi.org/10.1016/s0163-4453(99)90000-2

Jenson P (2019) In vitro cultivation Of protozoan parasites, 1st edn. CRC Press, Boca Raton

Krishnamurthy G, Vikram R, Singh SB, Patel N, Agarwal S, Mukhopadhyay G, Basu SK, Mukhopadhyay A (2005) Hemoglobin receptor in Leishmania is a hexokinase located in the flagellar pocket. J Biol Chem 280:5884-5891. https://doi.org/10. 1074/jbc.M411845200

Mahamoud A, Osman HA, Mansour D, el Harith A (2013) Successful substitution of fetal calf serum by human plasma for bulk cultivation of Leishmania donovani promastigotes. J Med Microbiol 62:11651169. https://doi.org/10.1099/jmm.0.052993-0

Merlen T, Sereno D, Brajon N, Rostand F, Lemesre JL (1999) Leishmania spp: completely defined medium without serum and macromolecules $(\mathrm{CDM} / \mathrm{LP})$ for the continuous in vitro cultivation of infective promastigote forms. Am J Trop Med Hyg 60:41-50. https://doi.org/10.4269/ajtmh.1999.60.41

Moreira D, Santarem N, Loureiro I, Tavares J, Silva AM, Amorim AM, Ouaissi A, Cordeiro-da-Silva A, Silvestre R (2012a) Impact of continuous axenic cultivation in Leishmania infantum virulence. PLoS Negl Trop Dis 6:e1469. https://doi.org/10.1371/journal.pntd.0001469

Moreira N, Vitoriano-Souza J, Roatt BM, Vieira PM, Ker HG, de Oliveira Cardoso JM, Giunchetti RC, Carneiro CM, de Lana M, Reis AB (2012b) Parasite burden in hamsters infected with two 
different strains of leishmania (Leishmania) infantum: "Leishman Donovan units" versus real-time PCR. PLoS One 7:e47907. https://doi.org/10.1371/journal.pone.0047907

Nicolle C (1908) Culture du parasite du bouton d'Orient, Gauthiers-Villars

Peters T (1996) All about albumin: biochemistry, genetics, and medical applications. Academic Press, San Diego, 1996 - Science - p 432

Reis LES, Brito RCF, Cardoso JMO, Mathias FAS, Aguiar Soares RDO, Carneiro CM, de Abreu Vieira PM, Ramos GS, Frezard FJG, Roatt BM, Reis AB (2017) Mixed formulation of conventional and pegylated meglumine antimoniate-containing liposomes reduces inflammatory process and parasite burden in Leishmania infantuminfected BALB/c mice. Antimicrob Agents Chemother 61. https:// doi.org/10.1128/AAC.00962-17

Roatt BM, Aguiar-Soares RD, Vitoriano-Souza J, Coura-Vital W, Braga SL, Correa-Oliveira R, Martins-Filho OA, Teixeira-Carvalho A, de Lana M, Figueiredo Gontijo N, Marques MJ, Giunchetti RC, Reis AB (2012) Performance of LBSap vaccine after intradermal challenge with L. infantum and saliva of Lu. longipalpis: immunogenicity and parasitological evaluation. PLoS One 7:e49780. https://doi. org/10.1371/journal.pone.0049780

Rodrigues Ide A, da Silva BA, dos Santos AL, Vermelho AB, Alviano CS, Dutra PM, Rosa Mdo S (2010) A new experimental culture medium for cultivation of Leishmania amazonensis: its efficacy for the continuous in vitro growth and differentiation of infective promastigote forms. Parasitol Res 106:1249-1252. https://doi.org/ 10.1007/s00436-010-1775-4

Sadigursky M, Brodskyn CI (1986) A new liquid medium without blood and serum for culture of hemoflagellates. Am J Trop Med Hyg 35: 942-944. https://doi.org/10.4269/ajtmh.1986.35.942

Santarem N, Cunha J, Silvestre R, Silva C, Moreira D, Ouellette M, Cordeiro DASA (2014) The impact of distinct culture media in
Leishmania infantum biology and infectivity. Parasitology 141: 192-205. https://doi.org/10.1017/S0031182013001388

Silva SC, Silva DF, Almeida TC, Perasoli FB, da Silva ATP, da Silva GN, Rezende SA (2018) Behavior of two Leishmania infantum strains-evaluation of susceptibility to antimonials and expression of microRNAs in experimentally infected J774 macrophages and in BALB/c mice. Parasitol Res 117:2881-2893. https://doi.org/10. 1007/s00436-018-5979-3

Siripattanapipong S, Boontanom P, Leelayoova S, Mungthin M, TanAriya P (2019) In vitro growth characteristics and morphological differentiation of Leishmania martiniquensis promastigotes in different culture media. Acta Trop 197:105039. https://doi.org/10. 1016/j.actatropica.2019.05.030

Smejkal RM, Wolff R, Olenick JG (1988) Leishmania braziliensis panamensis: increased infectivity resulting from heat shock. Exp Parasitol 65:1-9. https://doi.org/10.1016/0014-4894(88)90101-4

Steiger RF, Steiger E (1977) Cultivation of Leishmania donovani and Leishmania braziliensis in defined media: nutritional requirements. J Protozool 24:437-441. https://doi.org/10.1111/j.1550-7408.1977. tb04771.x

Sunter J, Gull K (2017) Shape, form, function and Leishmania pathogenicity: from textbook descriptions to biological understanding. Open Biol 7:170165. https://doi.org/10.1098/rsob.170165

Visvesvara GS, Garcia LS (2002) Culture of protozoan parasites. Clin Microbiol Rev 15:327-328. https://doi.org/10.1128/cmr.15.3.327328.2002

Publisher's note Springer Nature remains neutral with regard to jurisdictional claims in published maps and institutional affiliations. 